\title{
Lewis-base adducts of 9,10-dihydro-9,10-diboraanthracene: ditopic hydroboration reagents and a $B-N$ analogue of triptycene
}

\author{
Andreas Lorbach, Michael Bolte, Hans-Wolfram Lerner and Matthias Wagner*
}

\begin{abstract}
The dimethyl sulfide, pyridazine, or pyrazolide adducts of 9,10-dihydro-9,10-diboraanthracene are potent hydroboration reagents for terminal alkynes; the 1,2-diazene derivatives possess a paddle-wheel structure and are stabilised towards hydrolysis.
\end{abstract}

Hydroboration of carbon-carbon triple bonds is a powerful tool for the preparation of boron-doped $\pi$-conjugated organic macromolecules. ${ }^{1-4}$ For the following reasons, such compounds have promising potential as electron transporters and luminophores in organic light-emitting devices (OLEDs)..$^{5-7}$ (1) Due to the isoelectronic relationship between threecoordinate boron atoms and carbocations, the incorporation of boron into conjugated $\pi$-systems leads to similar electronic states as oxidative doping, but gives neutral materials. (2) In the presence of suitable $\sigma$-donors Do, the formation of B-Do adducts results in a shortening of the average $\pi$-conjugation length accompanied by a change in the electronic spectrum of the polymer. This is a highly useful effect, which can be exploited for the development of molecular switches and sensors.

While a broad variety of (hetero)aromatic diynes has already been employed in hydroboration polymerisation reactions, the choice of the boron component was long restricted mainly to mesitylborane, $\left(\mathrm{MesBH}_{2}\right)_{2}{ }^{1,2}$ Both for preparative reasons and in an attempt to improve the optoelectronic properties of the macromolecules, our group has recently introduced 9,10-dihydro-9,10-diboraanthracene as novel ditopic hydroboration reagent (1, Fig. 1). ${ }^{3} \mathbf{1}$ possesses a unique $\mathrm{B}-\mathrm{H}-\mathrm{B}$ bridged polymeric structure $[\mathbf{1}]_{n}$ in the solid state and is therefore insoluble in all common non-donor solvents. ${ }^{3}$ Nevertheless, the hydroboration polymerisation of $[1]_{n}$ with dialkynes was possible in THF under heterogeneous conditions and gave extended $\pi$-systems (e.g., 2; Fig. 1) showing an intense photoluminescence. ${ }^{3}$

In order to facilitate further development of this class of compounds, it would, however, be desirable to have soluble, highly crystalline low molecular weight derivatives of $\mathbf{1}$ that can easily be purified and used for further transformations in a

Institut für Anorganische Chemie, Goethe-Universität Frankfurt, Max-von-Laue-Strasse 7, D-60438 Frankfurt (Main), Germany. E-mail: Matthias.Wagner@chemie.uni-frankfurt.de Fax: + 496979829260

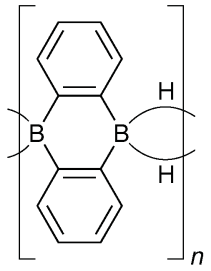

$[1]_{n}$

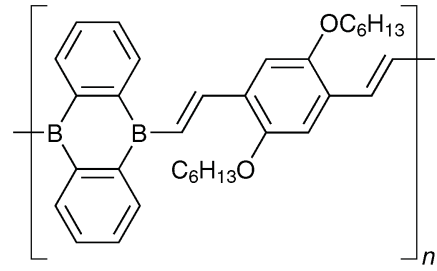

Fig. 1 The B-H-B bridged polymer $[\mathbf{1}]_{n}$ and the photoluminescent boron-doped $\pi$-conjugated system 2 prepared by hydroboration polymerisation of 1,4-diethynyl-2,5-bis(hexyloxy)benzene with $[\mathbf{1}]_{n}$ (THF, rt)

homogeneous liquid phase. All these requirements are met by the dimethyl sulfide (DMS) adduct 3 (Scheme 1), which is readily available when saturated solutions of 1 in dry DMS are cooled to $-78{ }^{\circ} \mathrm{C}$. The ${ }^{11} \mathrm{~B}$ NMR spectrum of 3 in $\mathrm{C}_{6} \mathrm{D}_{6}$ (DMS) solution shows one resonance at $28.1 \mathrm{ppm}(1.9 \mathrm{ppm})$, testifying to the presence of four-coordinate boron atoms. ${ }^{8}$ The better shielded boron nuclei in neat DMS compared to $\mathrm{C}_{6} \mathrm{D}_{6}$ indicate a dynamic $\mathrm{B}-\mathrm{S}$ association/dissociation equilibrium in the latter solvent. Yet the presence of only two equivalents of the Lewis base is still sufficient to prevent precipitation of polymeric $[\mathbf{1}]_{n}$. An X-ray crystal structure analysis of $\mathbf{3}$ is in line with the proposed diadduct structure (Fig. 2). The two DMS ligands are attached in a syn fashion to the 9,10-dihydro-9,10-diboraanthracene and the methyl groups are pointing away from the centre of the molecule. Consequently, the sulfur lone pairs closely approach each other $(\mathrm{S}(1) \cdots \mathrm{S}(2)=3.943(1) \AA)$. As in the parent compound $[\mathbf{1}]_{n}$, the borane backbone of $\mathbf{3}$ adopts a folded boat conformation with a dihedral angle between the two aryl rings of $\varphi=157^{\circ}\left([\mathbf{1}]_{n}: \varphi=134^{\circ}\right.$; in the corresponding pyridine diadduct, the pyridine ligands coordinate in an ant $i$ fashion and $\varphi$ equals $\left.180^{\circ}\right){ }^{3}$ Hydroboration of tert-butylacetylene with 3 in $\mathrm{C}_{6} \mathrm{D}_{6}$ proceeds rapidly and gives essentially quantitative yields (Scheme 1). An NMR spectroscopic investigation $\left({ }^{1} \mathrm{H},{ }^{11} \mathrm{~B}\right)$ of the solution prior to workup revealed that the vinylborane product was still coordinated by DMS. However, the ligands can easily be removed under reduced pressure to give donor-free $\mathbf{4}$, which is identical to a sample prepared with $[1]_{n}\left({ }^{1} \mathrm{H}\right.$ NMR spectroscopy; ${ }^{3}$ combustion analysis $)$.

The nature of $\mathbf{1}$ as a ditopic Lewis acid suggests applications as inverse chelator of bidentate Lewis bases. ${ }^{9}$ In the context of main-chain boron-doped polymers 2 , this approach offers several interesting perspectives: (1) since cooperative binding is possible, switching of optoelectronic polymer properties should be more efficient with bidentate than with monodentate donor molecules; (2) the four-coordination of the boron atoms 


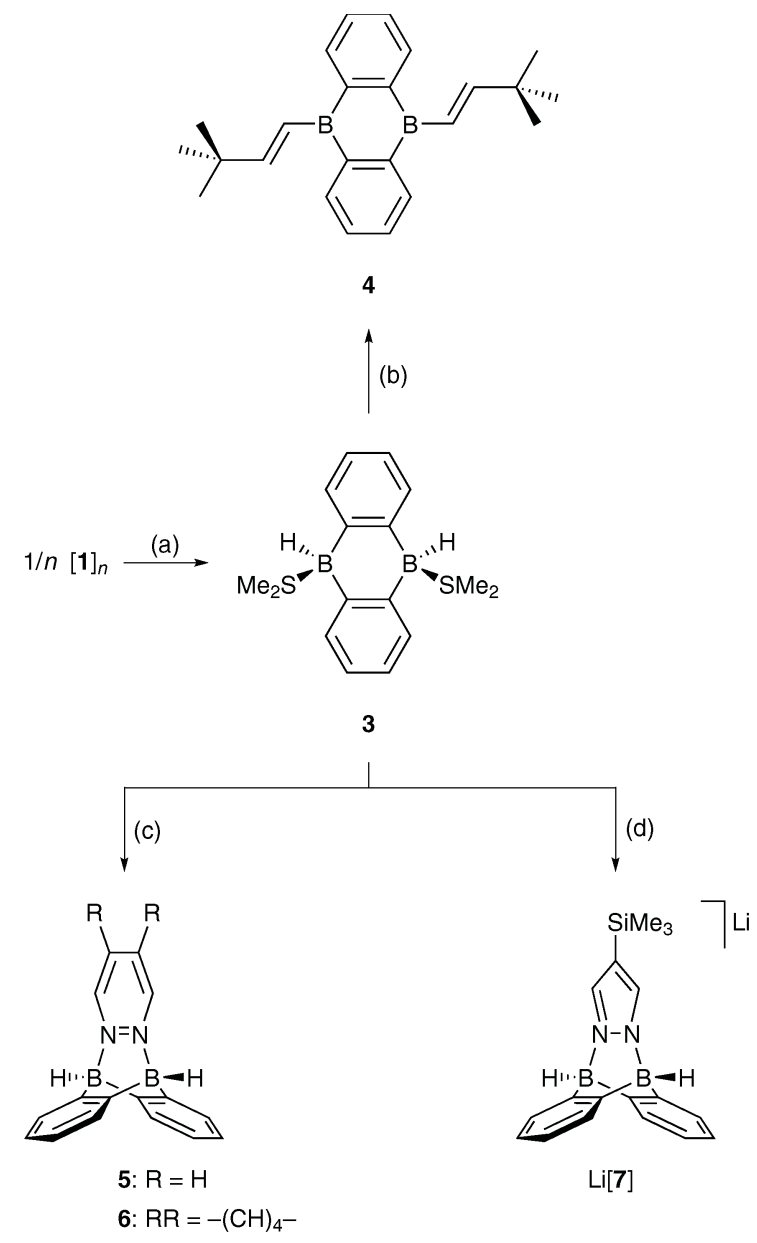

Scheme 1 Polymeric $[1]_{n}$ can be transformed into soluble 3, a convenient ditopic hydroboration reagent ( $c f$. 4). Paddle-wheel shaped adducts $(\mathbf{5}, \mathbf{6}$, and $\operatorname{Li}[7])$ are formed, when 3 is treated with 1,2diazenes. (a) DMS, rt; (b) $+5 t \mathrm{BuCCH}, \mathrm{C}_{6} \mathrm{H}_{6}$, rt; (c) $+1 \mathrm{C}_{4} \mathrm{H}_{2} \mathrm{~N}_{2} \mathrm{R}_{2}$, $\mathrm{C}_{6} \mathrm{H}_{6}, \mathrm{rt} ;(\mathrm{d})+1 \mathrm{Li}\left[\mathrm{C}_{3} \mathrm{H}_{2} \mathrm{~N}_{2}\left(\mathrm{SiMe}_{3}\right)\right], \mathrm{C}_{6} \mathrm{H}_{6} / \mathrm{THF}$, rt.

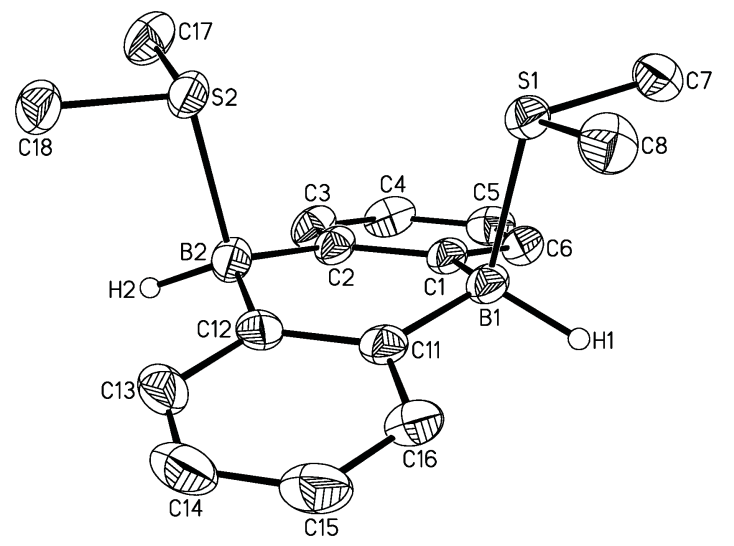

Fig. 2 Molecular structure and numbering scheme of compound 3; displacement ellipsoids are drawn at the $50 \%$ probability level; protons attached to carbon atoms are omitted for clarity. Selected (average) bond lengths $[\AA]$, atom $\cdots$ atom distances $[\AA]$, bond angles $\left[^{\circ}\right]$, and dihedral angles $\left[{ }^{\circ}\right]$ : $\mathrm{B}-\mathrm{C}=1.607(3), \mathrm{B}-\mathrm{S}=2.031(2), \mathrm{B}-\mathrm{H}=$ $1.19(2), \mathrm{B}(1) \cdots \mathrm{B}(2)=3.069(3), \mathrm{S}(1) \cdots \mathrm{S}(2)=3.943(1) ; \mathrm{C}-\mathrm{B}-\mathrm{C}=$ 115.0(2), C-B-S = 105.3(2), B-S-C = 103.9(1), C-S-C = 98.7(1); $\operatorname{Ar}(\mathrm{C}(1)) / / \operatorname{Ar}(\mathrm{C}(11))=157.0, \mathrm{~B}(1) \mathrm{C}(1) \mathrm{C}(11) / / \mathrm{B}(2) \mathrm{C}(2) \mathrm{C}(12)=33.8$. helps to stabilise the polymer chain with respect to oxidation or hydrolysis, e.g., during chain length determination by gel permeation chromatography or dynamic light scattering measurements; (3) the use of charged Lewis basic additives facilitates a polymer analysis by ESI or MALDI-TOF mass spectrometry. Given this background, we have treated the DMS adduct 3 with pyridazine and obtained the soluble compound 5 (Scheme 1), which is a $\mathrm{B}-\mathrm{N}$ analogue of triptycene. ${ }^{10}$ The ${ }^{11} \mathrm{~B}$ NMR spectrum of $\mathbf{5}$ shows one resonance at $5.3 \mathrm{ppm}$; the ${ }^{1} \mathrm{H}$ as well as the ${ }^{13} \mathrm{C} \mathrm{NMR}$ spectrum is characterised by only two signals for the phenylene rings and two signals for the pyridazine fragment (proton integral ratio $=2: 1$ ). All these spectroscopic features are in accordance with a highly symmetric molecular framework. Repeated attempts to grow X-ray quality crystals of $\mathbf{5}$ failed. We therefore replaced pyridazine by benzo $[d]$ pyridazine (Scheme 1) and confirmed the proposed molecular structure of the reaction product $\mathbf{6}$ by X-ray diffraction (details are given in the ESI $\dagger$ ). Apart from the two neutral 1,2-diazines mentioned above, 4-trimethylsilylpyrazolide has also been added to a solution of $\mathbf{3}$ in order to synthesise the anionic species [7] ${ }^{-}$(Scheme 1). In contrast to $\mathbf{5}$ and $\mathbf{6}$, which showed no molecular ion peaks in the $\mathrm{ESI}^{+/-}$mass spectrum (THF), the $\mathrm{ESI}^{-}$-MS of Li[7] in THF features exclusively one peak at $m / z=315.2\left([7]^{-}\right)$. This result strongly suggests that decoration of 2-type polymers with 4-trimethylsilylpyrazolide may indeed path the way to their (so far impractical) mass spectrometric characterisation. The X-ray crystal structure analysis of $\mathrm{Li}(\text { thf })_{4}[7] \cdot$ THF reveals a monomeric paddle-wheel framework for [7] ${ }^{-}$(Fig. 3) with an average B-N bond length

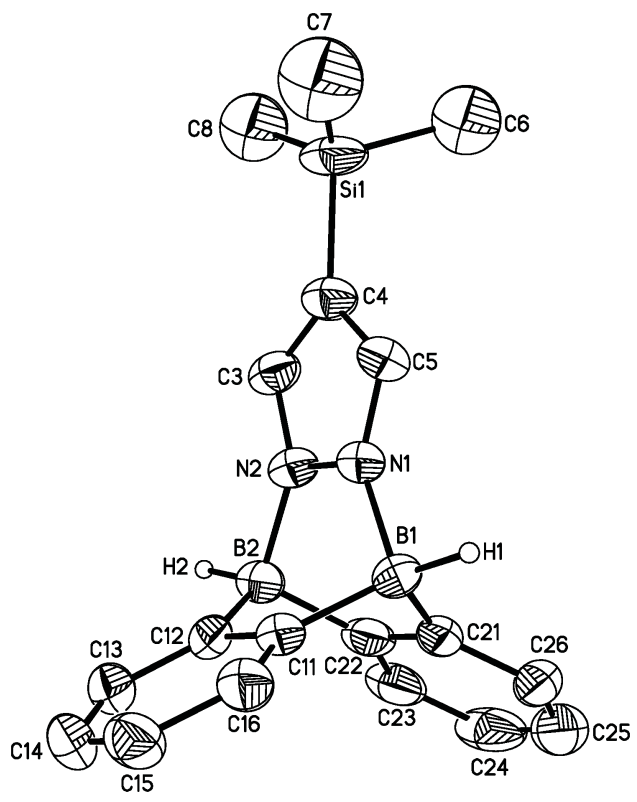

Fig. 3 Molecular structure and numbering scheme of the anion [7] ${ }^{-}$; displacement ellipsoids are drawn at the $50 \%$ probability level; protons attached to carbon atoms are omitted for clarity. Selected (average) bond lengths $[\AA]$, atom $\cdots$ atom distances $[\AA]$, bond angles $\left[{ }^{\circ}\right]$, and dihedral angles $\left[{ }^{\circ}\right]$ : $\mathrm{B}-\mathrm{C}=1.616(5), \mathrm{B}-\mathrm{N}=1.605(4), \mathrm{B}-\mathrm{H}=$ $1.19(5), \mathrm{B}(1) \cdots \mathrm{B}(2)=2.734(6) ; \mathrm{C}-\mathrm{B}-\mathrm{C}=105.8(3), \mathrm{C}-\mathrm{B}-\mathrm{N}=$ 103.2(3)， $\mathrm{B}-\mathrm{N}-\mathrm{N}=115.5(3) ; \operatorname{Ar}(\mathrm{C}(11)) / / \operatorname{Ar}(\mathrm{C}(21))=118.8$, $\mathrm{B}(1) \mathrm{C}(11) \mathrm{C}(21) / / \mathrm{B}(2) \mathrm{C}(12) \mathrm{C}(22)=84.6$. 
of 1.605(4) $\AA$ and an average B-N-N bond angle of $115.5(3)^{\circ}$ (cf. 6: $\left.\mathrm{B}-\mathrm{N}=1.643(3) \AA ; \mathrm{B}-\mathrm{N}-\mathrm{N}=114.3(2)^{\circ}\right)$. The folding of the 9,10-dihydro-9,10-diboraanthracene moiety in [7] $\left(\varphi=119^{\circ}\right)$ is slightly more pronounced than in $6\left(\varphi=127^{\circ}\right)$, but considerably more prominent than in $\mathbf{3}\left(\varphi=157^{\circ}\right)$.

Compound $\mathbf{3}$ is highly sensitive to air and moisture; in contrast, it takes several hours until the ${ }^{1} \mathrm{H}$ NMR spectra of solutions of $\mathbf{5}$ and Li[7] in wet THF show significant signs of decomposition. Both 5 and $\mathrm{Li}[7]$ are active hydroboration reagents towards tert-butylacetylene. The corresponding vinylboranes form as pyridazine/4-trimethylsilylpyrazolide adducts, which clearly indicates that the bridging of the two boron atoms by appropriate bidentate Lewis bases is not restricted to the parent compound $\mathbf{1}$, but should also be possible for 2-type polymers.

To summarise, in the context of main-chain boron-doped $\pi$-conjugated polymers we introduce the DMS adduct 3 of 9,10-dihydro-9,10-diboraanthracene $\mathbf{1}$ as a convenient ditopic hydroboration reagent for the transformation of alkynes into donor-free vinylboranes. Moreover, we show that various 1,2-diazenes can be used to bridge the boron atoms of 9,10-dihydro-9,10-diboraanthracene derivatives, which is of interest both for hydrolysis protection during polymer analysis and for the modulation of bulk physical polymer properties.
M.W. gratefully acknowledges financial support by the Beilstein Institute, Frankfurt/Main, Germany, within the research collaboration NanoBiC. A.L. wishes to thank the Fonds der Chemischen Industrie for a $\mathrm{PhD}$ grant.

\section{Notes and references}

1 N. Matsumi, K. Naka and Y. Chujo, J. Am. Chem. Soc., 1998, 120, 5112 .

2 N. Matsumi and Y. Chujo, Polym. J., 2008, 40, 77.

3 A. Lorbach, M. Bolte, H. Li, H.-W. Lerner, M. C. Holthausen, F. Jäkle and M. Wagner, Angew. Chem., Int. Ed., 2009, 48, 4584.

4 J. Chai, C. Wang, L. Jia, Y. Pang, M. Graham and S. Z. D. Cheng, Synth. Met., 2009, 159, 1443.

5 C. D. Entwistle and T. B. Marder, Angew. Chem., Int. Ed., 2002, 41, 2927.

6 F. Jäkle, Coord. Chem. Rev., 2006, 250, 1107.

7 M. Elbing and G. C. Bazan, Angew. Chem., Int. Ed., 2008, 47, 834.

8 H. Nöth and B. Wrackmeyer, Nuclear Magnetic Resonance Spectroscopy of Boron Compounds, in NMR Basic Principles and Progress, ed. P. Diehl, E. Fluck and R. Kosfeld, Springer, Berlin, 1978.

9 F. P. Gabbaï, A. Schier, J. Riede and M. J. Hynes, Chem. Commun., 1998, 897.

10 Compound $\mathbf{5}$ is one of very few examples of molecules in which a pyridazine ring bridges two boron atoms via $\mathrm{B}-\mathrm{N}$ bonds. For other examples see: (a) D. R. Martin, C. M. Merkel, J. U. Mondal and C. R. Rushing, Jr., Inorg. Chim. Acta, 1985, 99, 81; (b) D. J. H. Emslie, W. E. Piers and M. Parvez, Angew. Chem., Int. Ed., 2003, 42, 1251; (c) C. A. Jaska, D. J. H. Emslie, M. J. D. Bosdet, W. E. Piers, T. S. Sorensen and M. Parvez, J. Am. Chem. Soc., 2006, 128, 10885. 